\title{
Analysis of Electromagnetic Simulation Method for the Dielectric Lens Antenna
}

\author{
Farizah Ansarudin ${ }^{1,3}$, Tharek Abd. Rahman ${ }^{1}$, Yoshihide Yamada ${ }^{2 *}$, Nurul Huda \\ Abd Rahman ${ }^{2,4}$
}

${ }^{1}$ Universiti Teknologi Malaysia,

School of Electrical Engineering, Johor Bahru, 81310, MALAYSIA

${ }^{2}$ Universiti Teknologi Malaysia,

Malaysia-Japan International Institute of Technology, Kuala Lumpur, 54100, MALAYSIA

${ }^{3}$ Universiti Kebangsaan Malaysia,

Faculty of Engineering \& Built Environment, Selangor, 43650, MALAYSIA

${ }^{4}$ Universiti Teknologi MARA,

Faculty of Electrical Engineering, Shah Alam, Selangor, 40450, MALAYSIA

*Corresponding Author

DOI: https://doi.org/10.30880/ijie.2020.12.06.001

Received 4 October 2019; Accepted 28 May 2020; Available online 02 July 2020

\begin{abstract}
For the millimetre wave usage at 5G mobile communication system, a dielectric lens antenna will become a promising candidate for the base station antenna. In developing a dielectric lens antenna, antenna performance analysis by an electromagnetic simulator will be necessary. The structure of the proposed design is a hyperbolic dielectric lens antenna fed by a pyramidal horn at the operating frequency of $28 \mathrm{GHz}$. In this paper, the abilities of a commercial electromagnetic simulator FEKO are investigated. Simulation methods of GO and MoM solvers are shown for electric field distributions on the antenna aperture and far field radiation patterns. The simulation results show that the optimum gain of lens antenna based on GO and MoM method are 26.68 dBi and 27.24 dBi, respectively. Moreover, multi beam radiation patterns and suitable feed positions are clarified for wide scanning angle. Good performance for multi beam application by using MoM is shown based on the off-focus characteristic where the antenna gain is only slightly reduced from $0.51 \mathrm{dBi}$ to $4.03 \mathrm{dBi}$ for $5.71^{\circ}$ to $35.54^{\circ}$.
\end{abstract}

Keywords: Hyperbolic lens, refractive index, on-focus, off-focus, method of moment, geometrical optics

\section{Introduction}

In 5G mobile system, many new technologies are required such as millimetre wave (mm-wave), massive multiplein multiple-out (MIMO) technology [1], multi beam base station antenna, advanced multiple access and higher order modulation and coding. 5G requires a wide frequency band to provide high-speed communications. The current microwave frequency spectrum suffers from congestion and are not able to support the demand for higher data rate mobile smartphone users due to the limitation of spectrum at these frequencies. In developing new base station antennas, significant difference from the present system is the antenna size and its beam pattern. Multiple radiating beams with high gain performance are expected for the $5 \mathrm{G}$ base station as one radiating beam is assigned for one user only. 
This paper describes the use of dielectric lens antenna (DLA) for multi beam 5G base station application at $28 \mathrm{GHz}$ based on numerical method. At this frequency, DLA is selected by considering its ability to eliminate aperture blockage by the feed, excellent multi beam shapes, good scanning performance and acceptable in size and weight for many applications [2-4]. Besides, it can be easily contoured to any reasonable shapes to produce a specified beam pattern. There are two main categories of lens antenna depending on its application; either shaped lens antenna for collimating radiated energy or cylindrical and spherical for beam scanning with single or multiple feed possibility [4].Lens shaping techniques have been investigated by many researchers based on conventional geometrical optics (GO), transformation optics (TO), analytical method combining approximation of GO and physical optics (PO) as well as genetic algorithm depending on its applications [5-7]. The GO approach is fairly accurate for structures that are large compared to the wavelength of the radiation. In this paper, the numerical methods of spherical DLA for required beam pattern of on-focus and off-focus feed using MoM and GO method will be discussed.

\section{Lens Surface Equation}

The analysis of lens shaping had been discussed to ensure the accuracy of the design structure for multi beam application. The lens antenna structure and coordinate system are shown in Figure 1. The lens is symmetrical around the $\mathrm{z}$-axis. The $\mathrm{x}$-axis corresponds to the radial direction of the lens and z-axis corresponds to the longitudinal direction. The purpose of this paper is to clarify the suitable electromagnetic simulation method for a DLA. The simplest hyperbolic lens shape is selected. The feed is set at the focal point of $(0,0)$ coordinate. The lens inner surface is expressed by $(\mathrm{x}, \mathrm{z})$ coordinate. The distance from the focal point to the inner lens surface (S1) is expressed by $r$. The focal length, F and lens thickness, T are shown in Figure 1.

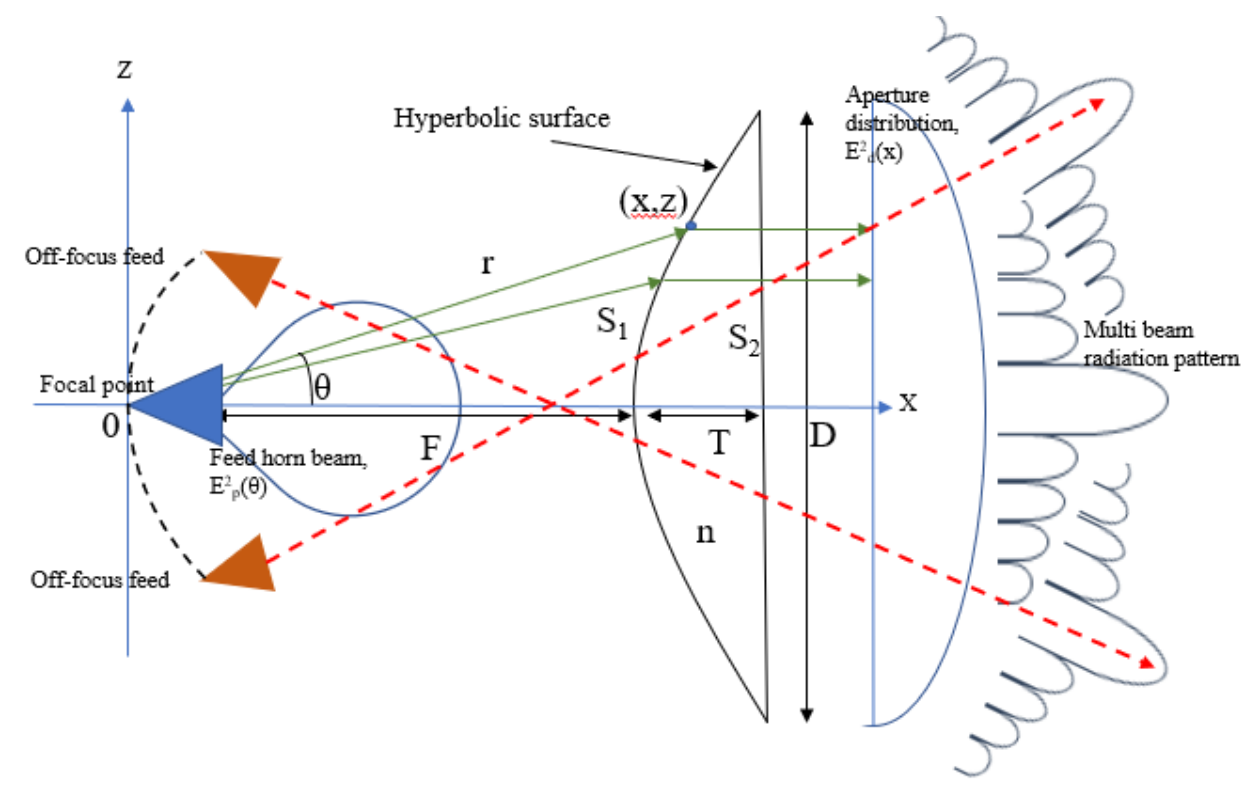

Fig. 1 - Hyperbolic lens antenna configuration and the concept of multi beam radiation pattern

When the inner surface is given by Eq. (1), (2) and (3), the refracted ray at the inner surface becomes parallel to the $\mathrm{x}$-axis [8]:

$$
\begin{aligned}
& r=\frac{(n-1) F}{n \cos \theta-1} \\
& x=r \cos \theta \\
& z=r \sin \theta
\end{aligned}
$$

where $\mathrm{n}$ is the refractive index of lens material and $\mathrm{F}$ is the focal length from radiating element to $\mathrm{S}_{1}$. For a given diameter, $\mathrm{D}$, the central thickness, $\mathrm{T}$ of the lens is expressed by: 


$$
T=\frac{1}{n+1}\left[F^{2}+\frac{(n+1) D^{2}}{4(n-1)}-F\right]
$$

\section{Electromagnetic Simulation Model}

\subsection{Dielectric Lens Antenna Structure}

Figure 2 shows the configurations of the hyperbolic surface of dielectric lens antenna in the electromagnetic simulator, FEKO. The lens is set symmetrical along y-axis. A pyramidal horn antenna is applied as a radiating element with maximum gain of $15.09 \mathrm{dBi}$. The lens diameter is about $10 \lambda$ with $28 \mathrm{GHz}$ frequency employed in the system. The ratio of focal length, $\mathrm{F}$ and lens diameter, $\mathrm{D}=100 \mathrm{is} \mathrm{F} / \mathrm{D}=1$. The hyperbolic lens surface is designed in FEKO based on Eq. (1), (2) and (3), respectively. The material used for dielectric lens antenna with permittivity value, $\varepsilon_{\mathrm{r}}=4$ and lens thickness, $\mathrm{T}$ is determined by Eq. (4). Table 1 shows the configuration of the dielectric lens antenna.

Table 1 - Dielectric Lens Antenna Configuration

\begin{tabular}{ll}
\hline Parameter & Dimension \\
\hline Diameter, D & $100 \mathrm{~mm}$ \\
Focal Length, F & $100 \mathrm{~mm}$ \\
Lens Thickness, T & $10.76 \mathrm{~mm}$ \\
Refractive index, $\mathrm{n}$ & 2 \\
Maximum angle, $\theta_{\mathrm{m}}$ & $24.29^{\circ}$ \\
\hline
\end{tabular}

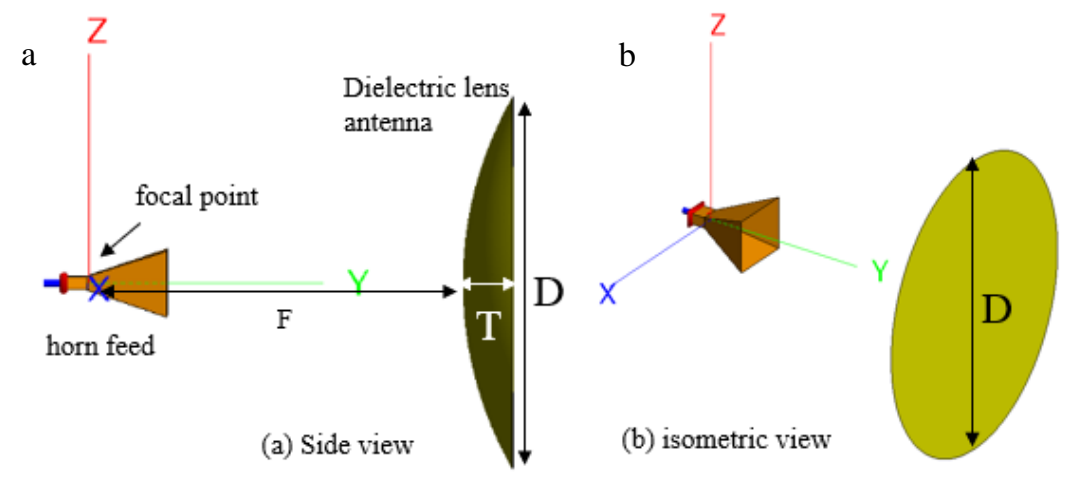

Fig. 2 - The dielectric lens antenna (a) side view; (b) isometric view

Table 2 shows the several coordinate points of the surface from origin, $\theta=0^{\circ}$ to maximum angle, $\theta_{\mathrm{m}}=24.29^{\circ}$. All 147 data points are calculated to design lens surface in FEKO using polyline curve.

Table 2 - Lens Surface Coordinates

\begin{tabular}{ccc}
\hline Angle, $\boldsymbol{\theta}\left({ }^{\circ}\right)$ & $\mathbf{y}$ & $\mathbf{z}$ \\
\hline 24.29 & 50 & 110.76 \\
20.19 & 39.36 & 107.01 \\
15.00 & 27.79 & 103.66 \\
10.04 & 17.98 & 100.16 \\
5.19 & 9.13 & 100.83 \\
0 & 0 & 100 \\
\hline
\end{tabular}




\subsection{Simulation Parameters}

The specification of the super computer in the Communication System Network Laboratory, MJIIT-UTM and the simulation parameters are shown in Table 3. Two simulation methods are conducted using Method of Moment (MoM) and hybrid simulation using Ray Launching-Geometrical Optics (RL-GO)-MoM for the whole antenna system. The horn feed with Perfect Electric Conductor (PEC) material is simulated based on MoM with mesh size of $\lambda / 12$. For dielectric lens antenna, mesh size of $\lambda / 5$ is employed for optimum simulation by MoM and GO method, respectively.

Table 3 - The Simulation Parameter

\begin{tabular}{lc}
\hline Parameter & Specification \\
\hline Computer & \\
Processor & Xeon $2.10 \mathrm{GHz}$ \\
Random Access Memory & $512 \mathrm{~GB}$ \\
Electromagnetic Software & FEKO 2018 \\
Lens Antenna & \\
refractive index, n & 2 \\
tan $\delta$ & 0.0004 \\
Mesh size & $\lambda / 5$ \\
Number of mesh & 66,112 \\
Feed Horn & \\
Mesh size & $\lambda / 12$ \\
Number of mesh & 18,532 \\
Calculation Time & \\
MoM & $1 \mathrm{H}$ \\
RL-GO and MoM (Hybrid) & $3 \mathrm{H}$ \\
\hline
\end{tabular}

\subsection{GO Method}

Multi beam characteristics based on ray tracing in this method is analysed using hybrid simulation, where two solvers, MoM and RL-GO are employed in performing the simulation of a feed horn and a DLA. The solver in FEKO that is used to produce ray tracing is RL-GO which is employed between the lens structure to the aperture plane. However, ray is not extended to the aperture plane unless other material is associated with the lens structure as shown in Figure 3. The rays can be refracted from surface 1 and surface 2 of the lens to the aperture plane by assigning an air region.

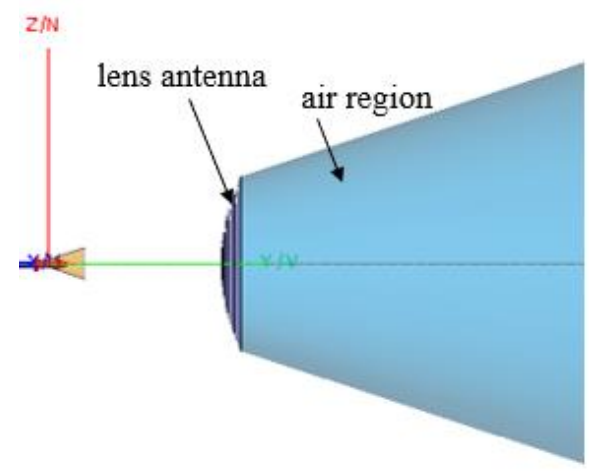

Fig. 3 - Lens antenna is associated with air region

Figure 4(a) shows all rays become parallel at on-focus feed after refracted from the lens surface. It shows that the lens concept of Figure 1 is verified clearly. In order to investigate the accuracies of GO method in FEKO, multi feed positions are simulated at $\theta_{\mathrm{F} 6}=35.54^{\circ}$ for off-focus feed as shown in Figure 4(b). It shows that the transmitted rays are almost parallel to the aperture plane and the accuracy of feed position is ensured. Figure 5 shows the radiation pattern simulated by GO method at $\theta_{\mathrm{F}}=0^{\circ}$ and $\theta_{\mathrm{F} 6}=35.54^{\circ}$, respectively. The results show that there is slight reduction in gain, and the difference is observed from $26.68 \mathrm{dBi}$ to $23.67 \mathrm{dBi}$ for $0^{\circ}$ to $35.54^{\circ}$. However, the beamwidth becomes larger from $\theta_{\mathrm{B}}=6.68^{\circ}$ to $\theta_{\mathrm{B}}=9.68^{\circ}$ which is clearly seen that the beam is distorted due to interruption of phase distribution. The concept of collimated rays occurred in on-focus and off-focus for each beam pattern is shown with parallel and almost parallel rays, respectively. 


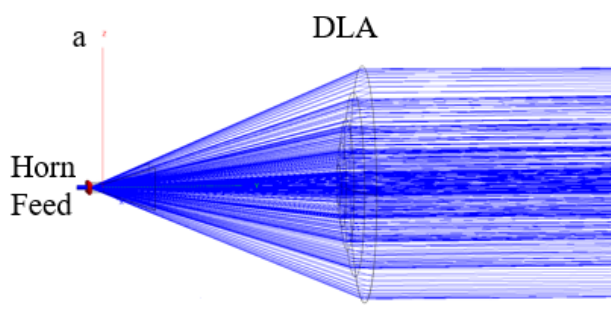

b

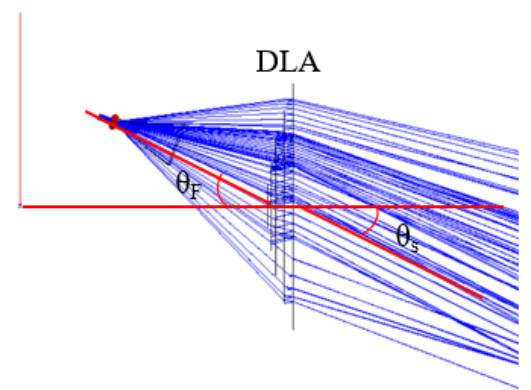

Fig. 4 - Ray tracing for different feed position (a) on-focus; (b) off-focus

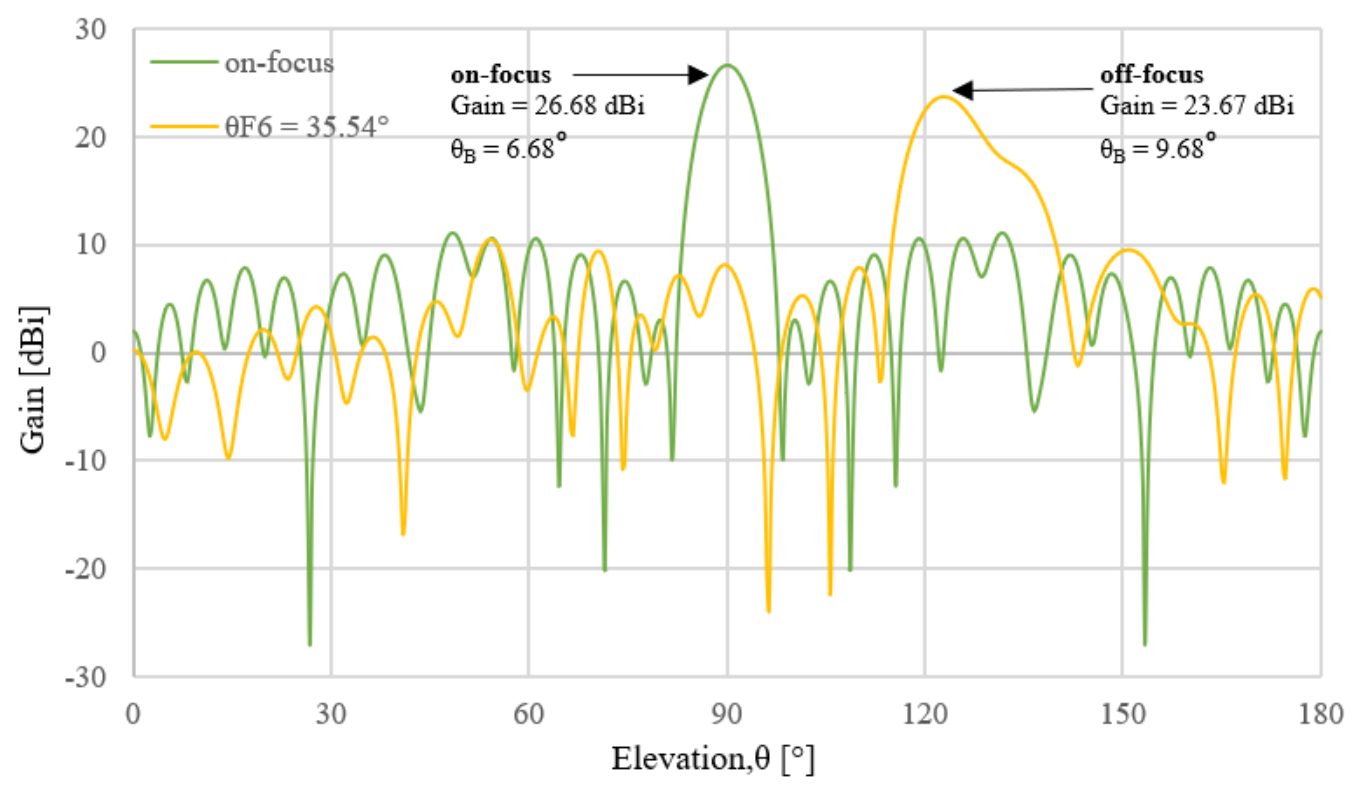

Fig. 5 - Radiation pattern by GO method

\subsection{MoM Method}

\subsubsection{Feed Horn Structure}

The horn is excited at the feed point by a waveguide source as shown in Figure 6 . At the focal point from lens surface, the phase centre is at horn neck about $21 \mathrm{~mm}$ inside from the horn aperture. The main specification in designing the feed horn is to obtain the correct radiation parameters [9-10], specifically around $\theta_{\mathrm{B}}=7.06^{\circ}$ beamwidth and low side-lobe level at the aperture plane that corresponds to $10 \lambda$ or $100.71 \mathrm{~mm}$. The horn dimension is designed based on Eq. (5) to obtain $10 \mathrm{~dB}$ edge level of lens at calculated beamwidth, $\theta_{\mathrm{B}}=34.39^{\circ}$.

$$
\theta_{B}=57 \frac{\lambda}{D}
$$

The simulated radiation pattern is almost symmetrical for E-plane and H-plane as shown in Figure 6 . The $3 \mathrm{~dB}$ beam width of each pattern is about $32.04^{\circ}$ and $32.98^{\circ}$, respectively. There is about $1.41 \mathrm{dBi}$ to $2.35 \mathrm{dBi}$ differences between the simulated and the theoretical beamwidth. These differences might not affect the main lobe shapes because the radiated power is very small, which is less than $10 \mathrm{dBi}$. The radiation angle to lens region of $\theta=2 \theta_{\mathrm{B}}$ for both E-plane and $\mathrm{H}$-plane are $\theta_{\mathrm{E}}=56.24^{\circ}$ and $\theta_{\mathrm{H}}=60.21^{\circ}$, respectively. However, the optimization of phase centre shall be considered for the feed position in obtaining a uniform phase. 

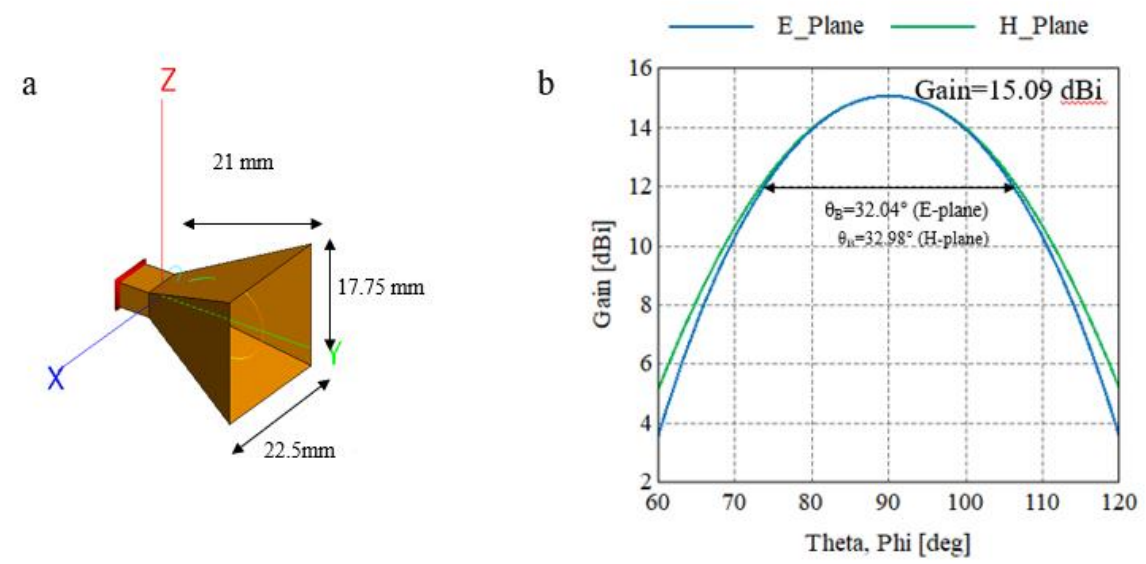

Fig. 6 - (a) Feed horn antenna; (b) Feed horn radiation pattern

\subsubsection{Aperture Distribution}

Antenna performance is investigated particularly according to its aperture distribution of field intensity and phase as shown in Figure 7 and Figure 8, respectively. The simulation of MoM method for the whole antenna system is employed for on-focus feed. From Figure 7, it is clearly seen that the edge level of the lens is $-10 \mathrm{~dB}$ and phase distribution is almost constant as shown in Figure 8.

a

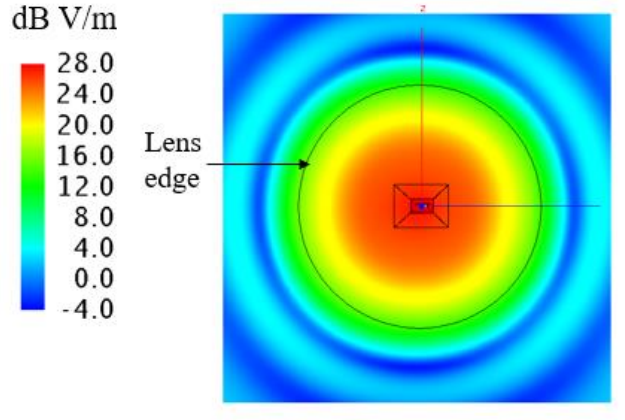

b

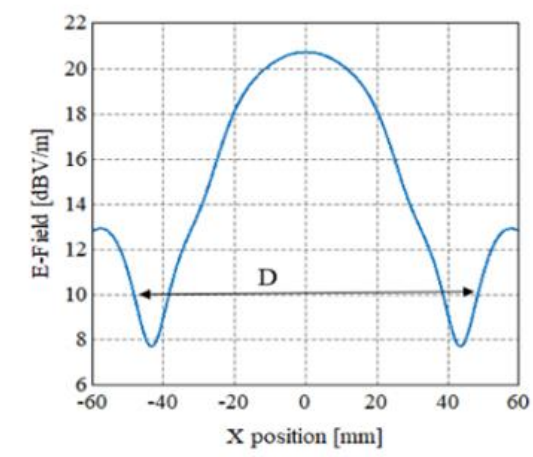

Fig. 7 - Electric field (a) intensity distribution; (b) amplitude distribution

a

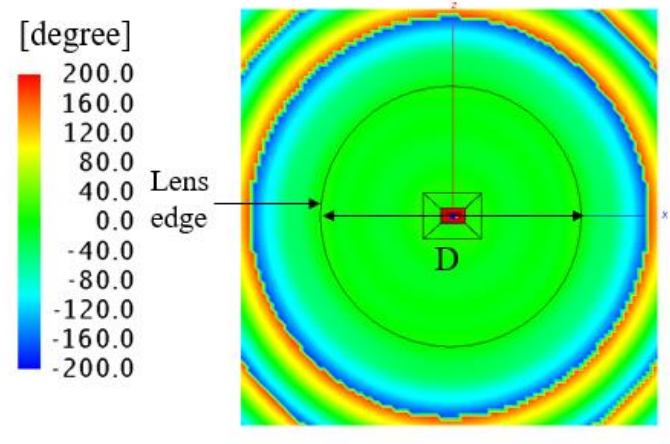

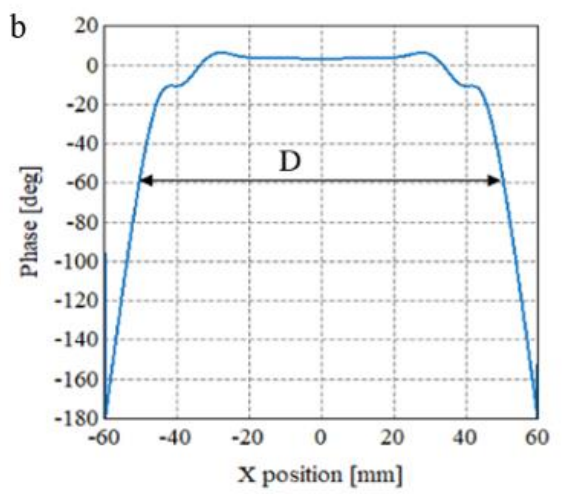

Fig. 8 - Electric (a) Phase contour; (b) Phase distribution

\subsubsection{Radiation Pattern}

The overall antenna system is simulated by electromagnetic software, FEKO using Method of Moment (MoM) and hybridization solver RL-GO with MoM at the operating frequency $28 \mathrm{GHz}$. The hybrid simulation is conducted for pyramidal horn (MoM) because of PEC material and dielectric lens antenna (RL-GO) as an insulation material for 
asymptotic method analysis at high frequency. The antenna is simulated for on-focus feed for $F / D=1$. All simulation results were compared with theoretical values of gain and beamwidth. Both theoretical gain and beamwidth are calculated using Eq. (6) and (7), respectively.

$$
\begin{aligned}
\theta_{B T} & =68.17 \frac{\lambda}{D} \\
G_{T} & =\left(\frac{\pi D}{\lambda}\right)^{2}
\end{aligned}
$$

The comparison of far field distribution at elevation angle between MoM and GO method is shown in Figure 9. The simulation results show that the optimum gain, Gs for GO and MoM method are 26.68 dBi and 27.24 dBi, respectively. The simulated gain, $\mathrm{G}_{\mathrm{S}}=27.24 \mathrm{dBi}$ for MoM corresponds to the aperture efficiency of $\eta=61.67 \%$ while GO of about $\eta=54.22 \%$ by comparing to the theoretical gain of $\mathrm{G}_{\mathrm{T}}=29.34 \mathrm{dBi}$ [9]. Moreover, values of simulated beam width $\theta_{\mathrm{BS}}=$ $7.01^{\circ}$ and $\theta_{\mathrm{BS}}=6.68^{\circ}$ for both methods were observed with theoretical values as tabulated in Table 4 . It is shown that the MoM method is more accurate for the gain and beam width when compared to GO due to full wave analysis for deriving rigorous solution in the electromagnetic simulator. The analysis of antenna performance can be extended in next measurement process for comparison studies between simulation and measurement results.

Table 4 - Beam width and gain for dielectric lens antenna

\begin{tabular}{cccc}
\hline $\begin{array}{c}\text { Theoretical } \\
\text { Beamwidth } \\
\theta_{\mathrm{BT}}\left(^{\circ}\right)\end{array}$ & $\begin{array}{c}\text { Simulated } \\
\text { beamwidth } \\
\theta_{\mathrm{BS}}\left(^{\circ}\right)\end{array}$ & $\begin{array}{c}\text { Theoretical } \\
\text { Gain, } \mathrm{G}_{\mathrm{T}} \\
(\mathrm{dBi})\end{array}$ & $\begin{array}{c}\text { Simulated } \\
\text { Gain, } \mathrm{G}_{\mathrm{S}} \\
(\mathrm{dBi})\end{array}$ \\
\hline $7.3^{\circ}$ & $7.01^{\circ}(\mathrm{MoM})$ & 29.34 & $27.24(\mathrm{MoM})$ \\
& $6.68^{\circ}(\mathrm{GO})$ & & $26.68(\mathrm{GO})$ \\
\hline
\end{tabular}

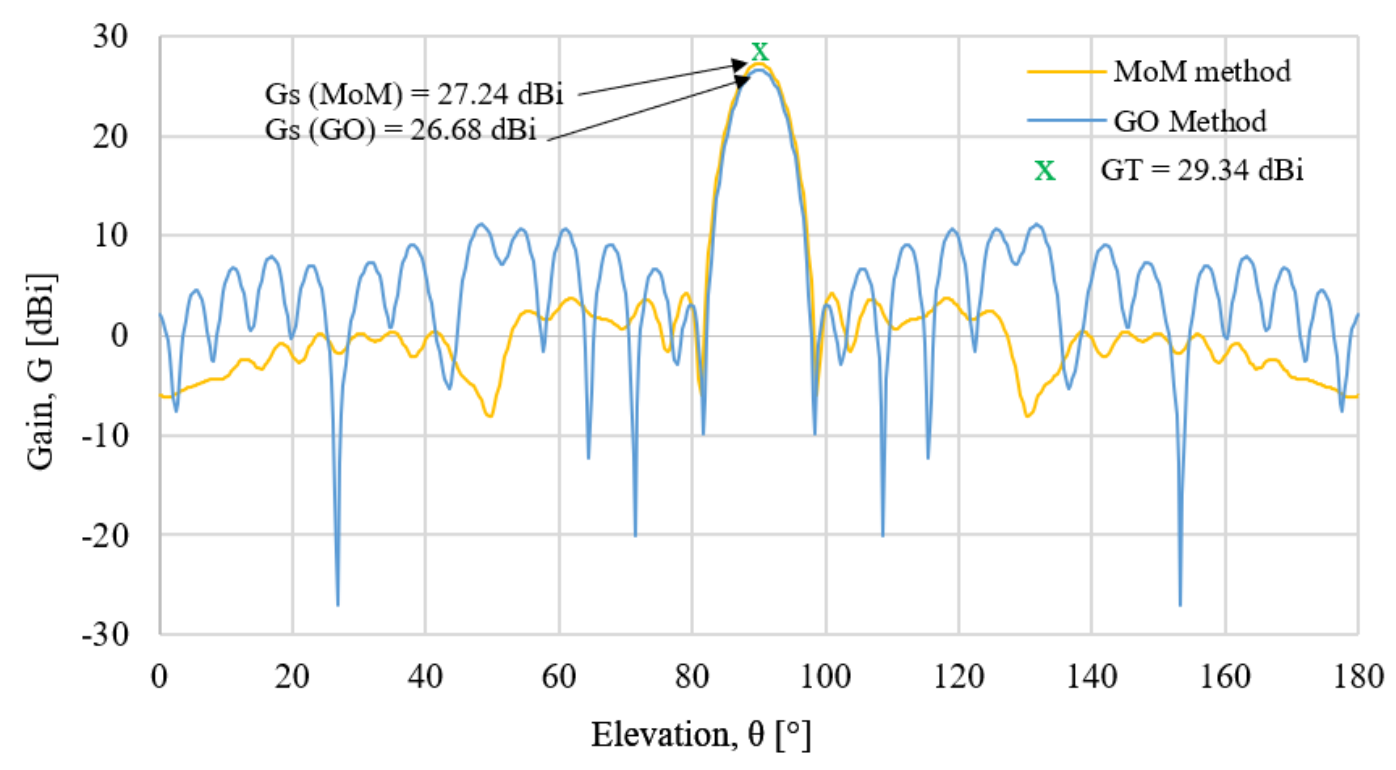

Fig. 9 - Radiation pattern by MoM and GO method 


\section{Multi Beam Characteristics}

\subsection{Feed Position}

The off-focus feeds for various angles have been employed at YZ plane as shown is Fig. 10. Feed points are typically shifted about $5^{\circ}$ to $8^{\circ}$ for each off-focus position. Previously, focal region was calculated through receiving mode ray tracing with caustic point as the best feed position [11-12]. In this paper, a simple linear regression is applied for offfocus feed determination. The optimum feed positions for off-focus feeds are shown in Figure 11 and Table 5. These positions are approximately expressed by Eq. (8).

$$
z=1.1619 y
$$

It is visibly seen that the feed position is linearly increased with increasing value of incident angle. In addition, shifted beam direction, $\theta_{\mathrm{s}}$ is smaller than the feed angle, $\theta_{\mathrm{F}}$ for F1 to F6. This condition encounters the principle of Snell's Law when the waves are passing through between two boundaries from free space to dielectric material. The locus of feed position, $\mathrm{R}(\mathrm{y}, \mathrm{z})$ from focal length, $\mathrm{F}$ can be derived as:

$$
R(y, z)=\frac{F-y}{\cos \theta_{F}}
$$

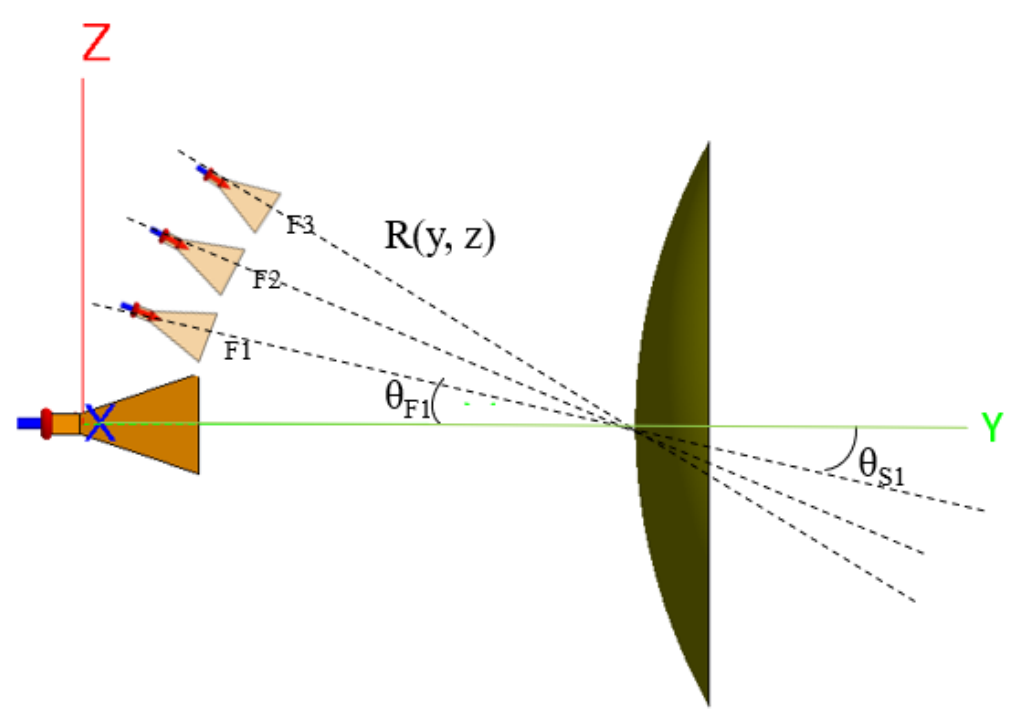

Fig. 10 - Off-focus beam scanning for various feed angle, $\theta_{F}$

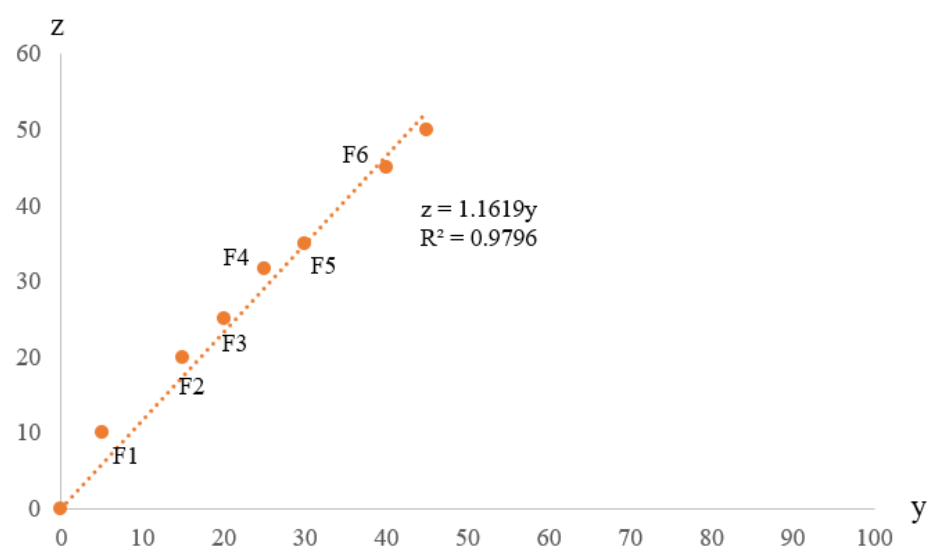

Fig. 11 - Linear regression for feed horn position with $\mathbf{R}^{2}=0.9796$ 
Table 5 - Feed positions for off-focus

\begin{tabular}{ccc}
\hline $\begin{array}{c}\text { Feed Number } \\
\text { (off-focus) }\end{array}$ & \multicolumn{2}{c}{ Feed Position } \\
\hline F1 & 5 & $\mathrm{z}$ \\
\hline F2 & 15 & 5.81 \\
F3 & 20 & 23.43 \\
F4 & 25 & 29.05 \\
F5 & 30 & 34.86 \\
F6 & 40 & 46.48 \\
\hline
\end{tabular}

\subsection{Off-Focus Radiation Patterns}

The off-focus beam characteristic is shown in Fig. 12 with scanning angle from $0^{\circ}$ to $35.54^{\circ}$ at $\mathrm{YZ}$ plane using MoM method. The gain is regularly reduced from $27.24 \mathrm{dBi}$ (on-focus) to $23.21 \mathrm{dBi}$ (F1-F6) when the incident angle of feed position is increased. It shows that the antenna performance using MoM for on-focus and off-focus is almost accurate for multibeam characteristics with gain reduction of about $0.42-3.8 \mathrm{dBi}$ from F1 to F6. The beam is slightly deteriorated (F6) at maximum scanning angle due to phase delay with the difference in gain of about 3.8dBi. Table 6 shows the antenna gain performance of on-focus and off-focus feed from F1 to F6 for scanning angle from $0^{\circ}$ to $35.54^{\circ}$.

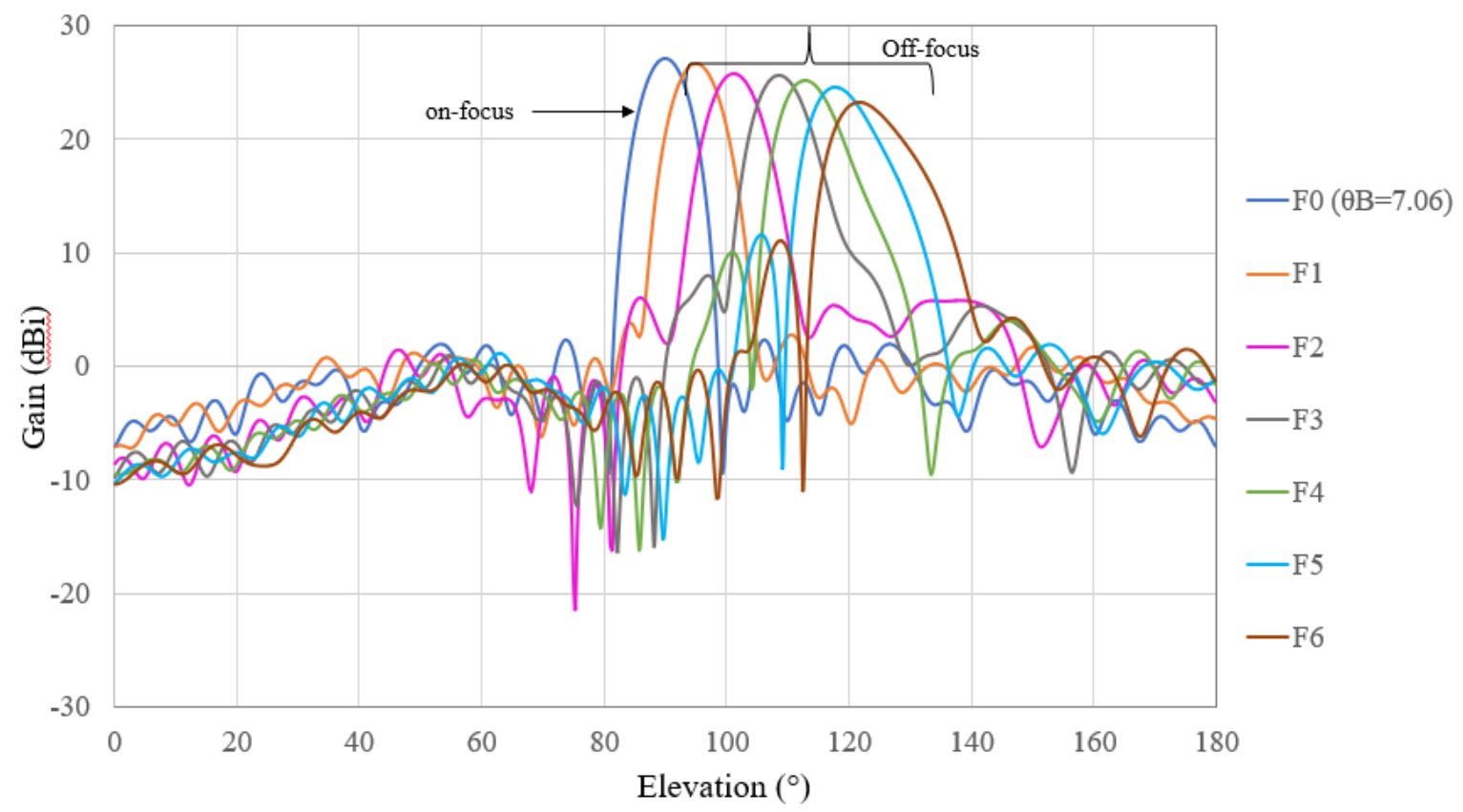

Fig. 12 - Radiation patterns for off-focus feeds at different angles

Table 6 - Off-focus feeds at different positions

\begin{tabular}{|c|c|c|c|c|c|}
\hline \multicolumn{2}{|c|}{ Feed Position } & $\begin{array}{c}\text { Feed Angle, } \\
\theta_{\mathrm{F}}\left({ }^{\circ}\right)\end{array}$ & $\begin{array}{c}\text { Shifted } \\
\text { Beam } \\
\text { Direction, } \\
\theta_{\mathrm{s}}\left(^{\circ}\right)\end{array}$ & $\begin{array}{c}\text { Beam } \\
\text { Width, } \\
\theta_{\mathrm{B}}\left(^{\circ}\right)\end{array}$ & $\begin{array}{l}\text { Gain } \\
(\mathrm{dBi})\end{array}$ \\
\hline on-focus & F0 & 0 & 0 & 7.06 & 27.24 \\
\hline \multirow{6}{*}{ off-focus } & $\Gamma \mathrm{F} 1$ & 5.71 & 5.08 & 7.28 & 26.59 \\
\hline & $\mathrm{F} 2$ & 13.87 & 11.08 & 7.75 & 25.77 \\
\hline & F3 & 21.80 & 18.57 & 7.91 & 25.62 \\
\hline & F4 & 26.56 & 22.83 & 8.40 & 25.17 \\
\hline & F5 & 30.47 & 27.89 & 9.33 & 24.53 \\
\hline & L F6 & 35.54 & 31.77 & 11.08 & 23.21 \\
\hline
\end{tabular}




\section{Conclusion}

Lens shape for hyperbolic surface simulated at $28 \mathrm{GHz}$ that corresponds to $10 \lambda$ diameter gives a promising beam width for base station installation. On-focus feed using hybrid method of RL-GO-MoM and MoM method provides optimum antenna performances. GO-MoM method shows that the concept of lens surface designing is ensured visibly because all rays become parallel to the aperture plane. However, multi feed position cannot be obtained accurately using ray tracing results in GO method due to the limitation of the 3D features of ray tracing plane view in FEKO. Besides, GO method is an approximation analysis for asymptotic technique for high frequency application. Conventionally, ray tracing technique for off-focus analysis can be developed by complete algorithm for computing the visibility between points [13]. The efficiency of simulated gain of MoM method is $61.67 \%$ accurate compared to GO method is only $54.2 \%$ between theoretical gain as calculated by (7). Beamwidth of MoM is slight closely to the theoretical values as calculated in (5) with difference angle of $0.29^{\circ}$ while GO $0.62^{\circ}$. The results of MoM show good aperture distribution of amplitude and phase at on-focus feed for near field distribution. The off-focus beam scanning used in MoM shows that the feed position between F1 to F6 is suitable for multibeam characteristics because of low reduction in gain of about $4.03 \mathrm{dBi}$ for scanning angle from $0^{\circ}$ to $35.54^{\circ}$. The multi feed position is ensured based on the analytical expression (8) where the beamwidth and gain obtained are not much reduced.

\section{Acknowledgement}

This work is carried out with the financial support from the Ministry of Higher Education, Malaysia for the HLP Scholarship and the Universiti Teknologi Malaysia Fundamental Research Grant Scheme (FRGS) with vote number 5 F046.

\section{References}

[1] Hong, W., Jiang, Z.H, Yu, C., Zhuo, C., P. Chen et al. (2017). Multibeam antenna technologies for 5G wireless Communications. IEEE Transaction on Antenna and Propagation, vol. 65, pp 6231-6249

[2] Thornton, J and Kao-Cheng, H. (2013). Modern Lens Antennas for Communications Engineering. John Wiley \& Sons

[3] Oscar, Q.T., Mahsa, E. and Fatemeh, G. (2018). Lens Antenna for 5G Communication Systems. IEEE Communications Magazine, July 2018, pp. 36-41

[4] Komljenovic, T. (2008). Lens Antenna-Analysis and Synthesis at mm-Waves. 50 ${ }^{\text {th }}$ International Symposium ELMAR, 10-12 Sept 2008, Croatia

[5] XiaoYang,Y. Z. XiaYing, Y. Yan, M. HuiFeng and C. TieJun. (2013). Three-dimensional large-aperture lens antennas with gradient refractive index". Science Chine Information Science. Vol. 56, pp. 1-12

[6] Tajima, Y. and Yamada, Y. (2006). Design of Shaped Dielectric Lens Antenna for Wide Angle Beam Steering. Electronics and Communications in Japan. Part 3. Vol. 89, No. 2, pp. 286-296

[7] Mozharovski, A.V, Artemenko, A.A, Mal'tsev, A.A, Maslennikov, R.O and Ssorin, V.N (2015). An Efficient Method for Calculating The Characteristics of The Integrated Lens Antenna on the Basis of The Geometrical Optic and Physical Optics Approximations. Radiophysics and Quantum Electronics. 18(6):pp. 443-453

[8] Lo, Y. T. and Lee, S.Y. (1993). Antenna Handbook Volume II. Van Nostrand Reinhold Company, Chapter 16, pp. 559

[9] Tajima, Y. and Yamada, Y. (2009). Simulations of Shaped Dielectric Lens Antenna by FEKO. The Applied Computational Electromagnetic Society Journal. Part 3. Vol. 24, No. 4, pp. 419-426

[10] Stutzman, W.L. and Thiele, G.A. (2013). Antenna Theory and Design ( ${ }^{\text {rd }}$ Edition). John Wiley \& Sons

[11] Kezuka, A., Tajima, Y., Yamada, Y. and Sasaki, S. (2004). Calculation of Wide Angle Radiation Pattern and Caustics of A Dielectric Lens Antenna by A Ray Tracing Method. IEICE Transaction on Electronics, Vol. 87(9), 1432-1440

[12] Sletter, C.J. (1988). Reflector and Lens. Artech House, pp. 235-239

[13] Tajima, Y., Yamada, Y., Sasaki, S. and Kezuka, A. (2014). Calculation of Wide Angle radiation Patterns and Caustics of a Dielectric lens Antenna by a Ray Tracing Method. IEICE Transaction on Electronics. Vol. E87-C, No. 9, pp. $1432-1440$ 